\title{
Theoretical and experimental study of the pulling force of jet bits in radial drilling technology
}

\author{
Guo Ruichang, Li Gensheng*, Huang Zhongwei, Tian Shouceng, \\ Zhang Xiaoning and Wu Wei \\ State Key Laboratory of Petroleum Resources and Prospecting, China University of Petroleum, Beijing 102249, China
}

\begin{abstract}
Radial drilling technology, of which the jet bit is the key device, is a research focus in the field of oil drilling and production. This paper establishes mechanical equations for jet bits and analyzes the hydroseal of backward jets in bottom holes. Meanwhile this paper establishes a mechanical equation for a high pressure hose and analyzes the axial force distribution. Laboratory experiments indicate that the flow rate, the angle between the backward nozzle axis and the jet bit axis, and the hole diameter are the major influencing factors; the generation of the pulling force is mainly due to the inlet pressure of the jet bit; the backward jets can significantly increase not only the pulling force but also the stability of jet bits. The pulling force would reach 8,376 N under experimental conditions, which can steadily pull the high-pressure hose forward.
\end{abstract}

Key words: Radial drilling, water jet, jet bit, depression effect, pulling force

\section{Introduction}

Radial drilling technology, which is also called ultra-short radius radial drilling technology, was developed over the last two decades. It is mainly applied to depleted reservoirs, fault block oil reservoirs, margin reservoirs and heavy oil reservoirs and has become a research focus in the field of oil drilling and production.

Ultra-short radius radial drilling technology is much different from conventional drilling technology. The key equipment of this technology mainly includes a jet bit, a high pressure hose, and a whipstock, as shown in Fig. 1. This technology completely depends on hydraulic energy to break rock. The flexible high pressure hose, which is strengthened by reinforcing layer in which steel cord is used as braided material, is used as the drilling pipe. The whipstock is small enough so that it can enter the main hole smoothly, avoiding underreaming. The high pressure hose is flexible enough to get through the whipstock, which has a track whose axis changes from vertical to horizontal in the ultra-short radius. This technology can drill several horizontal wells within the same pay zone up to one hundred meters long.

At present this technology has been used with good results in different places around the world, such as Argentina, Bolivia, and Russia (Cirigliano and Talavera Blacutt, 2007; Bruni et al, 2007; Ursegov et al, 2008). But there are still many difficulties in making the high pressure hose and jet bit move ahead smoothly, and controlling the

*Corresponding author. email: ligs@cup.edu.cn Received January 10, 2009

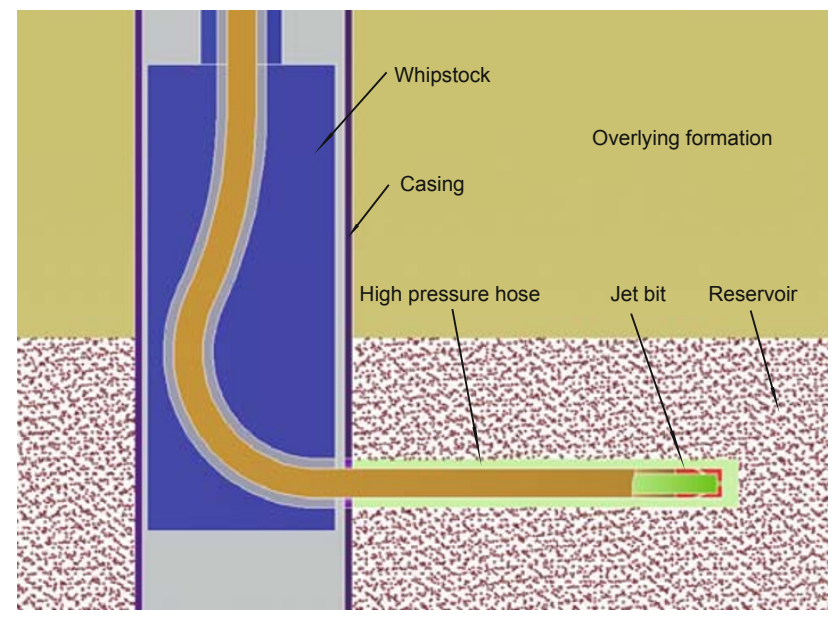

Fig. 1 Ultra-short radius radial drilling

hole trajectory, which has greatly hindered the development of this technology. Further research on the pulling force of the jet bit is important in resolving the difficulty how to make the high pressure hose move ahead smoothly. However, little research has been done on the generation of the pulling force of jet bit. Buset et al (2001) made a preliminary study and drew the conclusion that the complex bottom hole flow field can enhance the pulling force of the jet bit. The generation mechanism of the pulling force of the jet bit remains unclear. The authors established mechanical equations for the jet bit and high pressure hose, and analyzed the generation mechanism of the pulling force and the influence of the main factors. The theory was experimentally validated. 


\section{Working principle of the jet bit}

The working principle of the jet bit of the ultra-short radius radial drilling system is shown in Fig. 2. Compared with the commonly-used jet bit, the jet bit of the radial drilling system specially contains several backward nozzles. Therefore, the new type of jet bit can generate both forward and backward jets. The forward jets from the forward nozzles may be swirling or multiple jets, the main function of which is to break rock and make a hole with a diameter of several feet. The function of the backward jets is to increase the pulling force of the jet bit, and they also can enlarge the hole by scouring the hole wall while simultaneously removing cuttings.

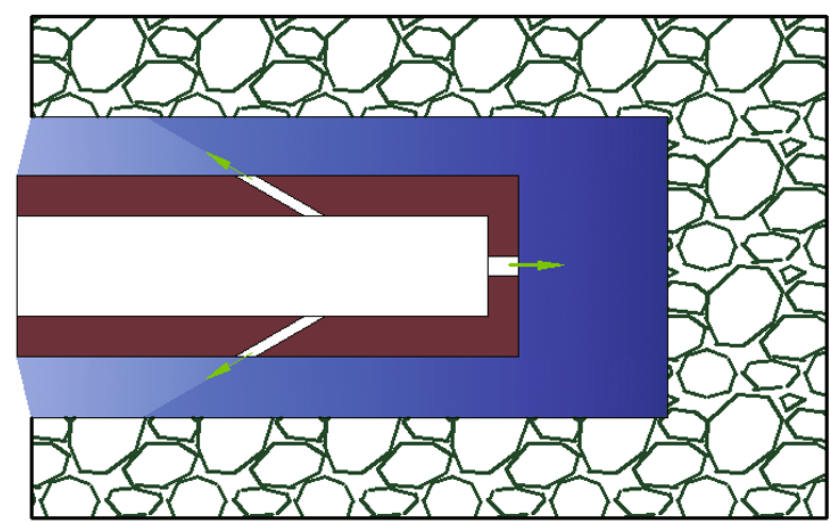

Fig. 2 Schematic diagram of working principle of the jet bit

The backward jets produce reverse thrusts, whose horizontal components are part of the pulling force, while the component forces in the radial direction are in balance (i.e. their resultant force is zero), in the ideal case that the jet bit is in the hole center, due to their symmetrical distribution around the circumference. However, when the jet bit is close to the lower side of hole wall, the radial component forces of reverse thrust produced by the lower side jets will increase, and then push the jet bit to the hole center until the resultant force of the radial component forces, gravity, and buoyancy of the jet bit becomes zero and the jet bit reaches a balanced state again. So the jet bit is usually suspended in the jetted hole when drilling, which makes hole trajectory control less difficult, and assures that a relatively straight, horizontal hole is drilled (Sunet al, 2006).

\section{Theory analysis of pulling force}

\subsection{Pulling force analysis of drilling nozzle}

According to the momentum theorem, the rate of momentum change in control volume is equal to the sum of all the external force applied to the control volume. As shown in Fig. 3, the momentum equation of the control volume in the $x$ direction is

$$
\iint_{A_{\text {in }}+A_{1}+A_{2}} \rho u_{x} u_{x} \mathrm{~d} A=\iint_{A_{z} \text { Totall }} \sigma_{x} \mathrm{~d} A+\iiint_{c v} F_{i} \mathrm{~d} V
$$

where the term on the left side of Eq. (1) represents the rate of momentum change $\Delta \bar{M}_{i}$ in the control volume in the $x$ direction; the first term on the right side of Eq. (1) represents the stress on the boundary of the control volume; and the second term is the weight component in the $x$ direction.

The $x$ direction represents the horizontal, and the weight component in this direction is zero. By integrating, Eq. (1) can be written as follows ( $\mathrm{Hu}, 2005)$ :

$$
\begin{aligned}
P_{\text {in }} A_{\text {in }}-P_{\text {out }} A_{\text {out }}-F_{\text {drag }} & =\rho Q_{\text {front }}\left(v_{\text {front }}-v_{\text {in }}\right) \\
& +n \times \rho Q_{\text {back }}\left(-v_{\text {back }} \cos \theta-v_{\text {in }}\right)
\end{aligned}
$$

Then the pulling force can be represented as

$$
\begin{aligned}
F_{\text {drag }}= & P_{\text {in }} A_{\text {in }}-P_{\text {out }} A_{\text {out }}-\rho Q_{\text {front }}\left(v_{\text {front }}-v_{\text {in }}\right) \\
& +n \times \rho Q_{\text {back }}\left(v_{\text {back }} \cos \theta+v_{\text {in }}\right)
\end{aligned}
$$

where $P_{\text {in }}$ and $P_{\text {out }}$ are the inlet and outlet pressures respectively of the jet bit, $A_{\text {in }}$ and $A_{\text {out }}$ are the inner and outer sectional areas of the jet bit, respectively; $Q_{\text {front }}$ and $Q_{\text {back }}$ are the flow rates of the forward and backward jets, respectively; $v_{\text {front }}$ and $v_{\text {back }}$ are the flow velocities of the forward and backward jets, respectively; $v_{\text {in }}$ is the flow rate in the hose; $\theta$ is the angle between the axis of the backward nozzle and the jet bit.

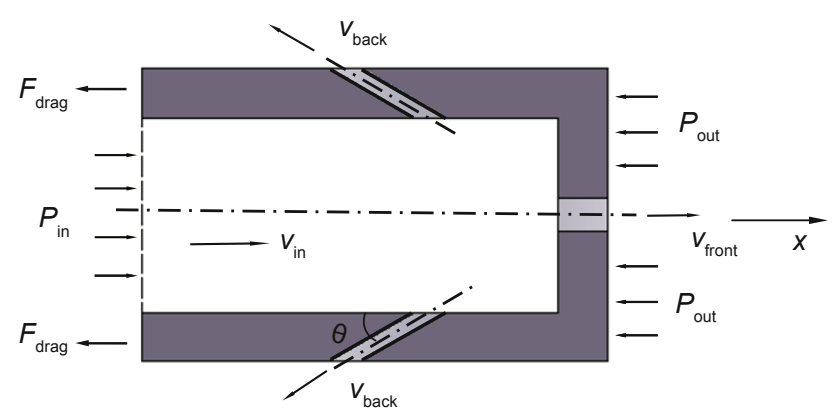

Fig. 3 Forces on the jet bit

The first two terms on the right side of Eq. (3) represent the forces generated by the inlet and outlet pressures of the jet bit. The last two terms are the rate of momentum change. According to Eq. (3), the pulling force increases when the angle $\theta$ decreases. When the angle $\theta$ is 0 , the pulling force reaches its maximum. Considering the limitation of manufacturing capability, the angle $\theta$ is generally $30^{\circ}-60^{\circ}$. The pulling force also increases with an increase in the flow rate of the backward jet. However, the whole flow rate of the jet bit is limited and a part of it has to be enough to ensure rock breaking, so the flow rate of backward jets cannot increase infinitely, so the pulling force due to the momentum change cannot be very large. In order to obtain a high speed jet, the jet bit pressure drop should be increased. When the pressure drop reaches $30-50 \mathrm{MPa}$, the produced the pulling force reaches $3,000-5,000 \mathrm{~N}$. So the pulling force of the jet bit is mainly generated by the high inner pressure. 
Due to the symmetrical distribution of the backward jets, the resultant force of the force generated by the backward jets in the radial direction is zero when the jet bit is located in the hole center. If there are no backward jets like the traditional jet bit, the jet bit is also subjected to a contact force except for gravity and buoyancy. The existence of the force generated by the backward jets changes this state. So the stability of the jet bit and high pressure hose is greatly enhanced.

\subsection{Force analysis of the high pressure hose}

The radial hole drilled by an ultra-short radius radial drilling system is horizontal, i.e. with a deviation angle of 90 degrees (Wang et al, 1999), so the forces on the high pressure hose, only when it is in the horizontal hole, are necessary to be analyzed (Fig. 4). The $x$ direction is horizontal. The governing equation is

$$
P_{1} A_{1}+F_{\text {pull }}=P_{2} A_{2}+F_{\mathrm{drag}}+F_{f}
$$

where $F_{\text {pull }}$ and $F_{\text {drag }}$ are the forces in the forward and backward directions, respectively; $P_{1}$ and $P_{2}$ are the pressures in the forward and backward directions respectively; $A_{1}$ and $A_{2}$ are the flow cross sectional areas at the inlet and outlet of the high pressure hose, respectively.

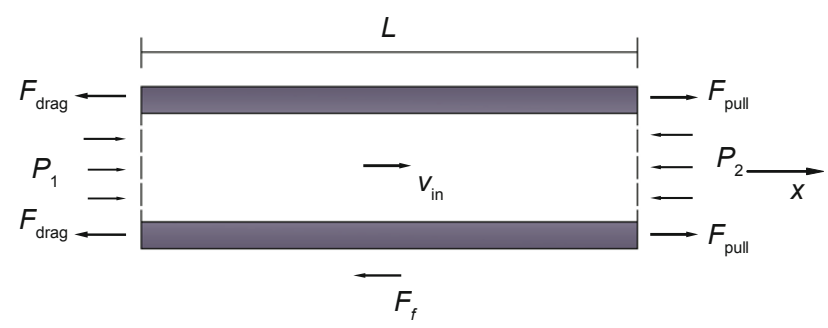

Fig. 4 Forces on a high pressure hose in a horizontal hole

In the $x$ direction, the pressure drop is mainly due to hydraulic friction along the high pressure hose, so Eq. (4) can be expressed as

$$
F_{\text {drag }}=F_{\text {pull }}-F_{f}+P_{1} A_{1}-P_{2} A_{2}=F_{\text {pull }}-F_{f}+\frac{2 f \rho v_{0}^{2} L}{d}
$$

where $f$ is the frictional coefficient; $v_{0}$ is the flow velocity in the high pressure hose; $L$ is the length of the high pressure hose; $d$ is the inner diameter of the high pressure hose.

The frictional drag $F_{f}$ between the high pressure hose and the hole wall can be calculated by

$$
F_{f}=\mu K_{\mathrm{b}} q_{\mathrm{p}} L
$$

where $\mu$ is the frictional coefficient between the high pressure hose and the hole wall; $K_{\mathrm{b}}$ is the buoyancy factor; $q_{\mathrm{p}}$ is the hose weight per unit length.

The hose weight per unit length is very small, varying from 0.4 to $0.5 \mathrm{~kg} / \mathrm{m}$, so that the frictional drag $F_{f}$ between the high pressure hose and the hole wall is small too. Meanwhile the thrust generated by the pressure drop along the high pressure hose is positive in the $x$ direction, so the force $F_{\text {drag }}$, which is equal to the pulling force of the next hose segment, won't decrease too much with the length of the high pressure hose and even will increase if the pressure drop gradient is large enough, which keeps the high pressure hose always in tension. The hose will still extend smoothly ahead in a steady straight state, even in an irregular hole.

\subsection{Depression effect of backward jets}

The high-speed water jets ejecting from the backward nozzles mixes with fluids in the annulus, carries them flowing backwards, and produces a steady low pressure zone acting as a "seal", which: 1) can reduce the chip hold-down effect to enhance penetration rate; and 2) can reduce backward pushing force generated by down hole pressure, resulting in an increase in the relative pulling force of the jet bit (Shen, 1998; Huang et al, 2008).

In order to calculate the pulling force, a differential pressure coefficient $\beta$ was introduced, which can be defined as follows

$$
\beta=\frac{\left(P_{\mathrm{o}}-P_{\mathrm{out}}\right)}{Q}=\frac{\Delta P}{Q}=f(Q, D)
$$

where $P_{\mathrm{o}}$ is the annular pressure behind the jet bit; $Q$ is the total flow rate; $D$ is the diameter of the hole.

The differential pressure coefficient $\beta$ is a function of the flow rate $Q$ and the hole diameter $D$, which represents the ability to generate a low pressure zone. Because the downhole flow fields are very complex, the differential pressure coefficient $\beta$ is difficult to calculate and has to be experimentally determined.

So the pressure in front of the jet bit can be represented as

$$
P_{\text {out }}=P_{\text {o }}-\beta Q
$$

The pulling force of the jet bit can be calculated by

$$
\begin{aligned}
F_{\text {drag }}= & P_{\text {in }} A_{\text {in }}-\left(P_{\mathrm{o}}-\beta Q\right) \times A_{\text {out }}-\rho Q_{\text {front }}\left(v_{\text {front }}-v_{\text {in }}\right) \\
& +n \times \rho Q_{\text {back }}\left(v_{\text {back }}+v_{\text {in }}\right)
\end{aligned}
$$

\section{Test equipment and method}

A jet bit with a single forward jet was used in the experiment. The pulling force was measured under experimental conditions: flow rate $0.1-2.0 \mathrm{~L} / \mathrm{s}$, ambient pressure $0 \mathrm{MPa}$, and hole diameter $30,40,50 \mathrm{~mm}$. The schematic equipment, as shown in Fig. 5, mainly includes a power and circulating system, experimental device, and a data acquisition system.

\section{Analysis of test results}

\subsection{Pressure drop at the jet bit}

A parabolic relationship between pressure drop and flow rate is shown in Fig. 6. High flow rate indicates a big pressure drop. Test results are basically identical with calculated results. When the flow rate was $1.0 \mathrm{~L} / \mathrm{s}$, the pressure drop of 


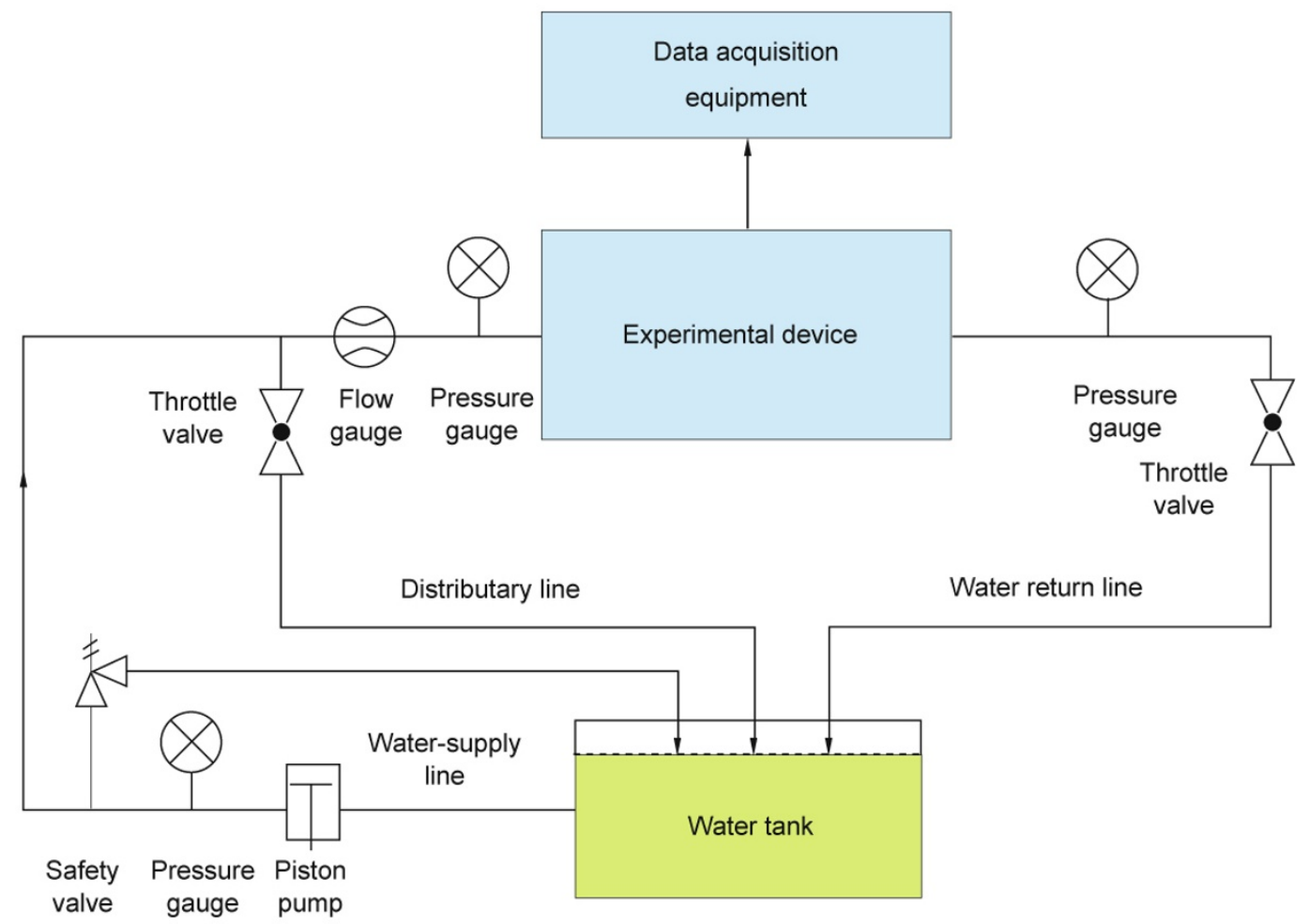

Fig. 5 Schematic diagram of testing device

the jet bit increased to $20 \mathrm{MPa}$. The force due to the pressure inside the jet bit was the main component of the whole pulling force. In order to obtain enough pulling force, the flow rate in field operation must be large enough.

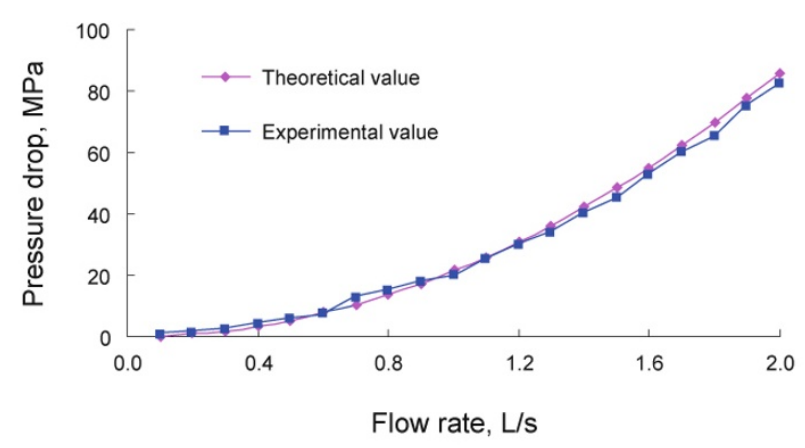

Fig. 6 Relationship between the pressure drop and the flow rate

\subsection{Downhole differential pressure}

Fig. 7 shows the relationship between the differential pressure coefficient $\beta$ and the flow rate in holes of different diameters. Test results showed that $\beta$ value increased approximately linearly with flow rate, and the larger the hole diameter, the smaller the rate of increase of the flow rate. So the higher the flow rate and the smaller the hole diameter, the larger the pressure differential between the zone in front of and behind the jet bit. Under experimental conditions, the $\beta$ value would reach $3.75 \times 10^{8}$, while the pressure differential would reach $0.75 \mathrm{MPa}$.

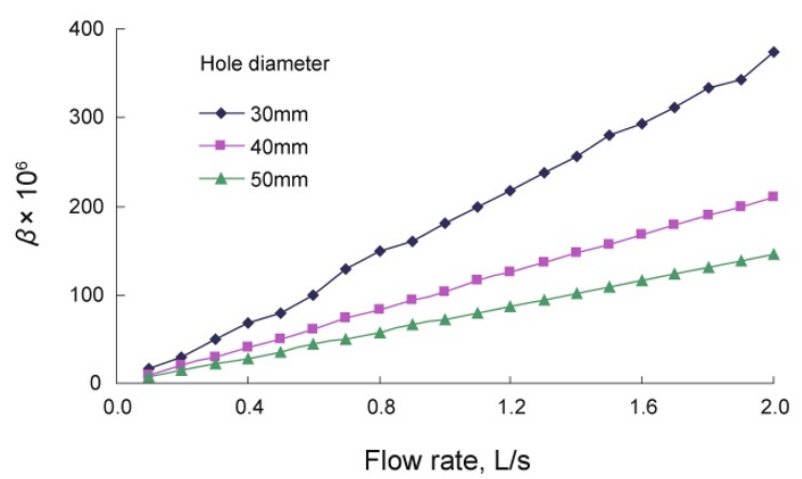

Fig. 7 Relationship between the pressure differential coefficient $\beta$ and the flow rate

\subsection{Pulling force of the jet bit in holes of different diameters}

Figs. 8-10 show the relationships between the pulling force of the jet bit and the flow rate in holes of different diameters. The red and the blue lines represent theoretical and experimental values respectively. The difference between theoretical and experimental values was within $3 \%$. When the flow rate was under $0.5 \mathrm{~L} / \mathrm{s}$, the pulling force remained small. When the flow rate was more than $1.0 \mathrm{~L} / \mathrm{s}$, the pulling force increased significantly. It would reach $8,000 \mathrm{~N}$ at a flow rate of $2.0 \mathrm{~L} / \mathrm{s}$. Because the high pressure flexible hose could only bear small axial force, the pulling force was the main factor in keeping the hose steady. 


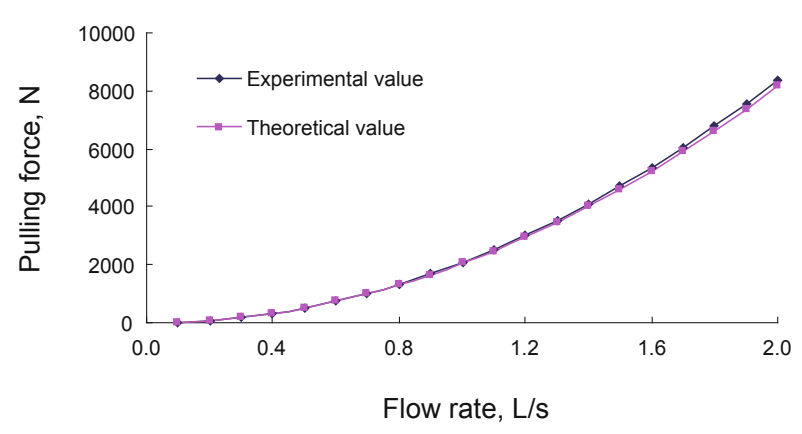

Fig. 8 Relationship between pulling force and flow rate, $D=30 \mathrm{~mm}$

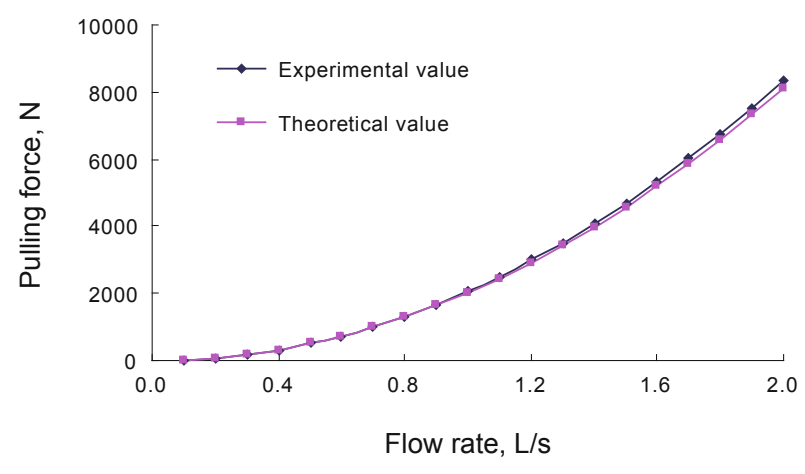

Fig. 9 Relationship between pulling force and flow rate, $D=40 \mathrm{~mm}$

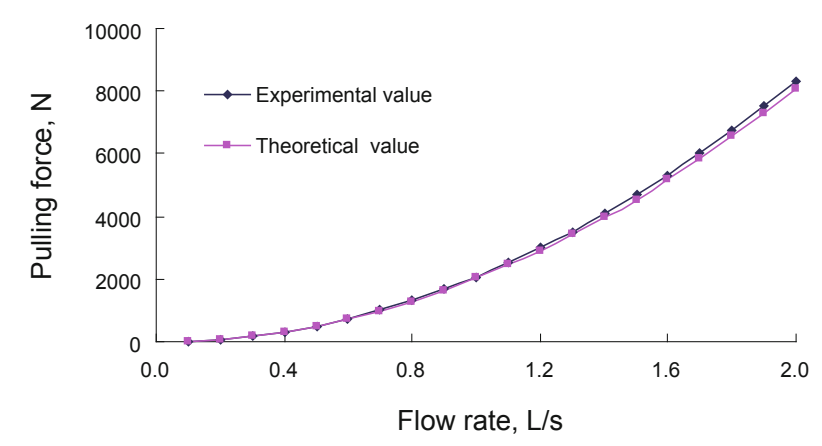

Fig. 10 Relationship between pulling force and flow rate, $D=50 \mathrm{~mm}$

\section{Conclusions}

1) The newly-designed jet bit can be used to break rock, remove cuttings, enlarge the drill-holes, and to produce pulling force. The depression effect of its backward jets from the backward nozzles reduces the chip hold-down effect, which helps enhance the penetration rate.
2) The pressure drop of the jet bit is the main factor in producing pulling force. The relationship between pressure drop and flow rate is parabolic.

3) The pressure differential coefficient $\beta$, characterizing the ability of the backward jets to produce depression region, increases linearly with the flow rate. The smaller the hole diameter, the greater the rate of increase of the flow rate. Under experimental conditions, $\beta$ value reaches $3.75 \times 10^{8}$ at a flow rate of $2.0 \mathrm{~L} / \mathrm{s}$.

4) Calculated and experimental results show that the pulling force reaches $8,000 \mathrm{~N}$ at a flow rate of $2.0 \mathrm{~L} / \mathrm{s}$.

\section{Acknowledgements}

This work was financially supported by High-tech Research and Development Program of China (No. 2007AA09Z315) and Doctoral Foundation of Ministry of Education of China (No. 20070425006). The authors are grateful for approval to publish.

\section{References}

Bruni M, Biassotti H and Salomone G. Radial drilling in Argentina. SPE Latin American and Caribbean Petroleum Engineering held in Buenos Aires, Argentina, April 15-18, 2007 (SPE paper 107382)

Buset P, Riiber M and Eek A. Jet drilling tool: cost-effective lateral drilling technology for enhanced oil recovery. SPE/ICoTA Coiled Tubing Roundtable held in Houston, Texas, March 7-8, 2001 (SPE paper 68504)

Cirigliano R and Talavera Blacutt J F. First experience in the application of radial perforation technology in deep wells. SPE Latin American and Caribbean Petroleum Engineering held in Buenos Aires, Argentina, April 15-18, 2007 (SPE paper 107182)

Huang Z W, Li G S,Tian S C, et al. Mechanism and numerical simulation of pressure stagnation during water jetting perforation. Petroleum Science. 2008. 5(1): 52-55

$\mathrm{Hu} \mathrm{Q}$ F. Analysis of jet pipe feeding in hydraulic deep penetrating operation. China Petroleum Machinery. 2005. 33(5): 7-9 (in Chinese)

Shen Z H. Water Jet Theory and Technology. Dongying: China University of Petroleum Press. 1998. 84-95 (in Chinese)

Sun N, Su Y N and Li G S. Progress in Drilling Engineering Technology. Beijing: Petroleum Industry Press. 2006. 120-122 (in Chinese)

Ursegov S, Bazylev A and Taraskin E. First results of cyclic steam stimulations of vertical wells with radial horizontal bores in heavy oil carbonates. SPE Russian Oil \& Gas Technical Conference and Exhibition held in Moscow, Russia, October 28-30, 2008 (SPE paper 115125)

Wang K S, Teng Q, Sun J H, et al. Theoretical analysis of lance propelling in coiled tubing water jet drilling. Journal of Beijing Institute of Machinery. 1999. 14(3): 21-24 (in Chinese)

(Edited by Sun Yanhua) 